\title{
In vivo and in silico screening for antimicrobial compounds from cyanobacteria
}

\author{
Selina Lenz ${ }^{1}$, Dorina Strieth ${ }^{1}$, and Roland Ulber ${ }^{1}$ \\ ${ }^{1}$ Technical University Kaiserslautern
}

February 8, 2022

\begin{abstract}
Due to the emerging rise of multi-drug resistant bacteria, the discovery of novel antibiotics is of high scientific interest. Through their high chemodiversity of bioactive secondary metabolites, cyanobacteria have proven to be promising microorganisms for the discovery of antibacterial compounds. These aspects make appropriate antibacterial screening approaches for cyanobacteria crucial. Up to date, screenings are mostly carried out using a phenotypic methodology, consisting of cyanobacterial cultivation, extraction, and inhibitory assays. However, the parameters of these methods highly vary within the literature. Therefore, the common choices of parameters and inhibitory assays are summarized in this review. Nevertheless, less frequently used method variants are highlighted, which lead to hits from antimicrobial compounds. In addition to the considerations of phenotypic methods, this review provides an overview of developments in the genome-based screening area, be it in-vivo using PCR technique or in-silico using the recent genome-mining method. Though, up to date these techniques are a lot less applied than phenotypical screening.
\end{abstract}

\section{Hosted file}

MB032021090446.pdf available at https://authorea.com/users/459569/articles/555795-in-vivoand-in-silico-screening-for-antimicrobial-compounds-from-cyanobacteria 